\title{
Investigation of Posture/Balance Disorder and Pd- related Pain by Using Diffusion Tensor Imaging and Transcranial Doppler
}

\section{Xiaoying Wang}

Second Hospital of Harbin Medical University

\section{Wenhui Guo}

Second Hospital of Harbin Medical University

\section{Yingying Zhang}

Second Hospital of Harbin Medical University

\section{Dan Liu}

Second Hospital of Harbin Medical University

\section{Qing Gao}

Second Hospital of Harbin Medical University

Limei Zhang ( $\square$ limeizhang1313@126.com )

Second Hospital of Harbin Medical University

\section{Kuang Fu}

Second Hospital of Harbin Medical University

\section{Research Article}

Keywords: Parkinson, posture/balance disorder, PD-related pain, white matter damage, diffusion tensor imaging, transcranial doppler

Posted Date: January 14th, 2022

DOI: https://doi.org/10.21203/rs.3.rs-1251083/v1

License: (a) (1) This work is licensed under a Creative Commons Attribution 4.0 International License. Read Full License 


\section{Abstract}

Background: Posture/balance disorder and pain are both present in Parkinson's patients, but their neural basis remain unclear. To investigate the central mechanism of posture/balance disorder and PD-related pain in Parkinson's disease by using diffusion tensor imaging (DTI) and tract-based spatial statistics (TBSS), combined with Transcranial Doppler (TCD).

Results: It was found that the dose of levodopa, UPDRS $\triangle$ and UPDRS $\triangle$ were higher value in the group with higher score of posture/balance. In the more severe posture/balance disorder group, the fiber bundles of the prefrontal cortex, anterior cingulate cortex and basal ganglia were more likely to be affected. In addition, the DTI parameter values of the three brain regions had a significant correlation with the parameter values of the corresponding arteries. In the analysis of PD-related pain, the white matter fiber bundles from the midbrain to the basal ganglia increased in patients with PD-related pain. There were no statistic difference in prevalence of PD-related pain was found between different groups according to posture/balance.

Conclusions: Posture and balance in PD were correlated with the severity of the disease and the dosage of compound levodopa. Posture and balance in PD were related to changes in the white matter integrity of the prefrontal cortex, anterior cingulate cortex and basal ganglia. The function of cerebral arteries had contributions to white matter integrity of these area and posture/balance. PD-related pain was positively correlated with sleep score. Patients with PD-related pain had an increase in the fiber projection from the midbrain to the basal ganglia. No relation was found between posture/balance disorder with PD-related pain.

\section{Background}

It is an effective method to explore the pathogenesis of Parkinson's disease (PD) in recent years to use diffusion tensor imaging (DTI) technique to trace the fiber and analyze the pathological changes of white matter in PD and its correlation with PD symptoms. Motor symptoms and non-motor symptoms of PD are related to each other and relatively independent. They may share a common pathological basis. But each has its own unique mechanism characteristics, for example, abnormal posture and associated pain in PD. Posture/balance disorder is one of the most important causes of disability in PD, it increases the risk of falls and lying in bed. The prevalence is twice as likely as age-matched older adults without PD ${ }^{[1]}$. Good posture and balance depend on integrity of brain fibers and an accurate regulation of tonic and phasic muscular activities, it is a complex integration of multi-sensory input ${ }^{[2]}$. It was reported postural instability/gait difficulty (PIGD) patients have more severely affected micro structural changes within the substantia nigra and the globus pallidus. In addition, degeneration in frontal white matter is connected to PIGD symptoms in $\mathrm{PD}^{[3]}$. Balance training may be related to changes in the gray matter of the bilateral anterior cingulate gyrus (ACC) ${ }^{[4]}$, and improve the posture and gait disorder. 
Chronic pain in PD may partly be due to the abnormal posture. The ACC plays an important role in the perception of pain. Otherwise, an activation of the prefrontal cortex (PFC) in animal experiments can induce aversion and anxiety in rats with neuropathic pain, and aggravate spontaneous pain ${ }^{[5]}$. This overlaps pathologically with posture/balance disorder in the brain. In order to further understand the central mechanism of posture/balance disorder and PD-related pain, and explore whether there is correlation between them. We relied on the advanced processing of DTI, combined with transcranial doppler to reveal the structural changes and activation of brain regions related to posture/balance disorder and pain. we analyzed the changes of white matter fibers in ACC, PFC and basal ganglia, and observed the changes of blood flow in the corresponding brain regions. Simultaneously tracked the fiber bundles from the midbrain to these brain regions.

\section{Materials And Methods}

\subsection{Participants}

Twenty patients were recruited in the outpatients office of the $2^{\text {nd }}$ hospital of Harbin Medical University. They were diagnosed with primary PD according to MDS criteria (2016), age from 45-80 years old. Excluding the disease of severe depression, schizophrenia, space of fear, cognitive dysfunction, severe cardiopulmonary renal dysfunction, there are metal or pacemakers in the body.

\subsection{Clinical assessment}

The patients were evaluated with MDS-UPDRS (2017), Hamilton Anxiety Scale (HAMA), Hamilton Depression Scale (HAMD), Mini-Mental State Examination (MMSE), Pittsburgh Sleep Quality Index (PSQI), and medications were recorded.

\subsection{Recording and analysis of cerebral blood flow}

Transcranial Doppler (TCD) was performed using a commercially available TCD device (SONARA Digital TCD, Childs Nerv Syst CareFusion, Middleton, WI).Blood flow velocities were record bilaterally in the middle cerebral arteries (MCA), anterior cerebral artery (ACA) and internal carotid arteries (ICA). The pulsation index $(\mathrm{PI})$ and resistance index (RI) were calculated automatically with each recording.

\subsection{MRI data acquisition}

DTI was performed using a 3.0T GE MRI scanner. DTI data was obtained using a single-shot echo-planar imaging sequence with the parameters as follows (non-collinear directions $=30, T R=1000 \mathrm{~ms}, T E=84 \mathrm{~ms}$, matrix $=128 \times 128, F O V=240 \times 240 \mathrm{~mm}^{2}$, slice thickness $=4 \mathrm{~mm}$, b-value $=1000 \mathrm{~s} / \mathrm{mm}^{2}$ ). High-resolution T1and T2-weighted images were acquired for each participant to exclude the possibility of recessive lesions.

Diffusion data was processed and analyzed using functional MRI of the brain (FMRIB) software library (FSL version 6.0) from the Oxford University Centre. Eddy current distortion and head motion were 
corrected using the Eddy Correct tool. Then the b0 image of each subject was skull-stripped with the brain extraction tool. After spatial normalization of the diffusion tensor data, maps of the fractional anisotropy $(F A)$, mean diffusivity (MD), radial diffusivity (RD), and axial diffusivity (AD) values were calculated from the transformed tensors.

For tract-based spatial statistics (TBSS), the FA data was non-linearly registered to a standard space (the FMRIB58-FA-space template) and then affine-aligned to the $1 \times 1 \times 1$ Montreal Neurological Institute (MNI 152) space. After converting into MNI space, the mean FA image was created and thinned to a skeleton with FA value threshold at 0.2. Each subject's aligned FA data was then projected onto this skeleton. Next, $A D, R D$, and $M D$ images were also aligned into $M N I$ space and projected onto the mean FA skeleton using the non-FA protocol in TBSS. According to the existing research and brain atlas, extracted PFC, ACC, and basal ganglia as the region of interest (ROI) (see Fig.1) to make a mask file, extracted the brain area of interest and the average value of the skeletal index. Diffusion toolkits was used to track fiber bundles from the midbrain to the target brain area.

\subsection{Statistical analysis}

All data were analyzed using SPSS 26 (IBM Corp., Armonk, NY, USA). The measurement data was tested by chi-square, and the count data was statistically compared using student's t test or analysis of variance (ANOVA). The data that does not fit the normal distribution was assessed with Mann-Whitney $U$ test. Spearman correlation analysis was used to evaluate the correlation between DTI numerical parameters and relevant data. The difference was statistically significant $(P<0.05)$.

\section{Results}

\subsection{Posture/balance disorder group data}

\subsubsection{Clinical features}

The sample comprises 20 participants with PD, of whom 10 subjects were men (50\%) \average age (65.3 \pm 10.75 years old $) ; 10(50 \%)$ women with average age of $(62.9 \pm 9.31$ years old). The score of posture/balance was $3.12+3.13$ according to MDS-UPDRS (3.12 posture balance, 3.13 posture). Nine patients with the total score of 2 and lower were labled as "Group1". Eleven patients with the total score of 3 and more were labled as "Group2". Group2 patients showed higher scores of UPDRS-II and UPDRS-III, being accepted higher levodopa dose, comparing with Group 1(See Table1). Moreover, Group2 possessed higher average age, a higher score of HAMA, HAMD and PSQI than those of Group1, but they showed no statistical difference.

Table1 Clinical characteristics between the groups according to posture/balance. 


\begin{tabular}{|lllll|}
\hline & Group 1(n=9) & Group 2(n=11) & T/Z & $P$ \\
\hline Levodopa Dose & $177.78 \pm 164.1$ & $404.55 \pm 155.65$ & -3.164 & 0.005 \\
\hline UPDRSII & $6.22 \pm 4$ & $13.55 \pm 7.11$ & -2.776 & 0.012 \\
\hline UPDRSIII & $19.27 \pm 7.2$ & $29.22 \pm 10.56$ & -2.401 & 0.027 \\
\hline MMSE & $29(28-29.5)$ & $29(27-29)$ & -0.926 & 0.539 \\
\hline Disease duration & $6.22 \pm 3.03$ & $5.91 \pm 2.98$ & 0.232 & 0.819 \\
\hline Age & $60.56 \pm 7.54$ & $67 \pm 10.9$ & -1.5 & 0.151 \\
\hline HAMA & $3(1.5-17)$ & $10(3-25)$ & -0.761 & 0.446 \\
\hline HAMD & $9.67 \pm 9.54$ & $13.55 \pm 9.77$ & -0.893 & 0.384 \\
\hline PSQI & $7.33 \pm 5.59$ & $8.1 \pm 4.28$ & 0.258 & 0.74 \\
\hline
\end{tabular}

The value of data that conforms to the normal distribution is displayed as the mean \pm standard deviation, and the value of the data that does not conform to the normal distribution is displayed as the median(quartile). MMSE: Mini-Mental State Examination; HAMA: Hamilton Anxiety Scale; HAMD: Hamilton Depression Scale; PSQI: Pittsburgh sleep quality index.

\subsubsection{DTI information}

Fiber tracking was performed by DTI, Group2 showed lower value of FA and FA skeleton in ACC, PFC, and basal ganglia. For the parameters of RD and RD skeleton, MD and MD skeleton, $A D$ and $A D$ skeleton, there were higher value of the first five terms in ACC; and higher value of all parameters in PFC; higher value of $R D$ skeleton, $M D$ skeleton and $A D$ skeleton in basal ganglia $(P<0.05)$. They overlap with the difference in FA. There were no significant difference between the two groups in the comparison of the $A D$ skeleton of $A C C$, the $A D, M D$, and $R D$ of the basal ganglia $(P>0.05)$. ( Table 2$)$

\section{Table 2: The difference between the groups in DTI index}




\begin{tabular}{|c|c|c|c|c|}
\hline & Group $1(n=9)$ & Group 2(n=11) & $\mathrm{T} / \mathrm{Z}$ & $P$ \\
\hline ACC FA & $0.178(0.168-0.181)$ & $0.16099(0.15-0.177)$ & -2.697 & 0.007 \\
\hline ACC FA skeleton & $0.502 \pm 0.044$ & $0.454 \pm 0.049$ & 2.295 & 0.034 \\
\hline ACC RD & $0.00101 \pm 0.0001$ & $0.0012 \pm 0.0002$ & -2.903 & 0.009 \\
\hline ACC RD skeleton & $0.00055 \pm 0.00005$ & $0.00064 \pm 0.000097$ & -2.483 & 0.023 \\
\hline ACC MD & $0.0011 \pm 0.0001$ & $0.00129 \pm 0.00017$ & -2.887 & 0.01 \\
\hline ACC MD skeleton & $\begin{array}{l}0.0008(0.00079- \\
0.00083)\end{array}$ & $0.00085(0.00082-0.0009)$ & -2.089 & 0.037 \\
\hline$A C C A D$ & $0.00128 \pm 0.00012$ & $0.00148 \pm 0.00017$ & -2.843 & 0.011 \\
\hline ACC AD skeleton & $\begin{array}{l}0.00129(0.00123- \\
0.00134)\end{array}$ & $\begin{array}{l}0.00131(0.00126- \\
0.00142)\end{array}$ & -1.178 & 0.239 \\
\hline PFC FA & $0.121(0.116-0.127)$ & $0.112(0.107-0.121)$ & -2.545 & 0.011 \\
\hline PFC FA skeleton & $0.4(0.378-0.411)$ & $0.3695(0.338-0.39)$ & -2.621 & 0.009 \\
\hline PFC MD & $0.00073 \pm 0.00006$ & $0.000815 \pm 0.00008$ & -2.58 & 0.019 \\
\hline PFC MD skeleton & $\begin{array}{l}0.000576(0.0007- \\
0.00079)\end{array}$ & $\begin{array}{l}0.00081(0.00079- \\
0.00086)\end{array}$ & -2.773 & 0.006 \\
\hline PFC AD & $0.00085 \pm 0.00007$ & $0.00093 \pm 0.00008$ & -2.589 & 0.019 \\
\hline PFC AD skeleton & $\begin{array}{l}0.0011(0.00107- \\
0.00113)\end{array}$ & $\begin{array}{l}0.00115(0.00112- \\
0.00116)\end{array}$ & -2.393 & 0.017 \\
\hline PFC RD & $0.00067 \pm 0.00006$ & $0.00076 \pm 0.00008$ & -2.564 & 0.019 \\
\hline PFC RD skeleton & $\begin{array}{l}0.00057(0.00056- \\
0.0006)\end{array}$ & $0.00063(0.0006-0.0007)$ & -2.545 & 0.011 \\
\hline Basal ganglia FA & $0.247(0.236-0.256)$ & $0.2235(0.212-0.242)$ & -2.089 & 0.037 \\
\hline $\begin{array}{l}\text { Basal ganglia FA } \\
\text { skeleton }\end{array}$ & $0.419(0.407-0.448)$ & $0.397(0.379-0.413)$ & -2.317 & 0.02 \\
\hline Basal ganglia AD & $0.00146 \pm 0.0001$ & $0.00162 \pm 0.0002$ & -1.743 & 0.098 \\
\hline $\begin{array}{l}\text { Basal ganglia AD } \\
\text { skeleton }\end{array}$ & $0.00117(0.001-0.0012)$ & $0.00122(0.0012-0.00127)$ & -2.241 & 0.025 \\
\hline Basal ganglia MD & $0.0012 \pm 0.00012$ & $0.00135 \pm 0.0002$ & -1.778 & 0.092 \\
\hline $\begin{array}{l}\text { Basal ganglia MD } \\
\text { skeleton }\end{array}$ & $\begin{array}{l}0.00078(0.00076- \\
0.0008)\end{array}$ & $0.00085(0.0008-0.00088)$ & -2.925 & 0.003 \\
\hline Basal ganglia RD & $0.00106 \pm 0.0001$ & $0.0012 \pm 0.0002$ & -1.794 & 0.09 \\
\hline $\begin{array}{l}\text { Basal ganglia RD } \\
\text { skeleton }\end{array}$ & $\begin{array}{l}0.00059(0.00057- \\
0.00062)\end{array}$ & $\begin{array}{l}0.00066(0.00062- \\
0.00069)\end{array}$ & -3.001 & 0.005 \\
\hline
\end{tabular}


The value of data that conforms to the normal distribution is displayed as the mean \pm standard deviation, and the value of the data that does not conform to the normal distribution is displayed as the median (quartile).The skeleton is a white matter skeleton, and differences between groups indicate significant results. If the difference of the corresponding data parameters is not on the skeleton, the meaning is not as good as the skeleton result. ACC: anterior cingulate cortex; PFC: prefrontal cortex; FA: fractional anisotropy; MD: mean diffusivity; RD: radial diffusivity; AD: axial diffusivity.

\subsubsection{TCD ultrasound parameters between the two groups}

Both groups of patients according to posture/balance disorder were detected with TCD. Group 2 showed significantly higher value of the PI and RI of bilateral MCA, ACA and ICA than those of the Group $1(P<0.01)$. But there were no statistic difference in the peak and mean velocities in MCA, ACA and ICA. (Table 3)

\section{Table3: The difference between the groups in the blood supply}

\begin{tabular}{|lllll|}
\hline & Group $1(\mathrm{n}=9)$ & Group $2(\mathrm{n}=11)$ & $\mathrm{T} / \mathrm{Z}$ & $\mathrm{P}$ \\
\hline Left MCA PI & $0.925 \pm 0.058$ & $1.069 \pm 0.18$ & -2.543 & 0.025 \\
\hline Left MCA RI & $0.568 \pm 0.021$ & $0.618 \pm 0.065$ & -2.427 & 0.031 \\
\hline Right MCA PI & $0.87(0.815-1)$ & $1.28(0.99-1.35)$ & -3.003 & 0.005 \\
\hline Right MCA RI & $0.545(0.525-0.595)$ & $0.69(0.62-0.71)$ & -3.202 & 0.002 \\
\hline Right ACA PI & $0.834 \pm 0.14$ & $1.19 \pm 0.2$ & -4.286 & 0.001 \\
\hline Right ACA RI & $0.53 \pm 0.056$ & $0.66 \pm 0.067$ & -4.27 & 0.001 \\
\hline Left ACA PI & $0.85(0.81-0.92)$ & $1.29(0.95-1.46)$ & -3.309 & 0.002 \\
\hline Left ACA RI & $0.55 \pm 0.03$ & $0.67 \pm 0.078$ & -5.043 & $<0.001$ \\
\hline Right ICA terminal end PI & $0.89 \pm 0.098$ & $1.177 \pm 0.22$ & -3.426 & 0.003 \\
\hline Right ICA terminal end RI & $0.55 \pm 0.04$ & $0.65 \pm 0.07$ & -3.34 & 0.004 \\
\hline Left ICA terminal end PI & $0.92 \pm 0.074$ & $1.13 \pm 0.17$ & -3.152 & 0.006 \\
\hline Left ICA terminal end RI & $0.57 \pm 0.028$ & $0.64 \pm 0.056$ & -3.382 & 0.004 \\
\hline PI of left ICA siphon segment & $0.925(0.89-0.95)$ & $1.2(1.01-1.35)$ & -3.423 & 0.001 \\
\hline RI of left ICA siphon segment & $0.57(0.555-0.58)$ & $0.66(0.6-0.71)$ & -3.397 & 0.001 \\
\hline PI of right ICA siphon segment & $0.92 \pm 0.1$ & $1.23 \pm 0.22$ & -3.674 & 0.002 \\
\hline RI of right ICA siphon segment & $0.57 \pm 0.04$ & $0.67 \pm 0.07$ & -3.627 & 0.002 \\
\hline
\end{tabular}


The value of data that conforms to the normal distribution is displayed as the mean \pm standard deviation, and the value of the data that does not conform to the normal distribution is displayed as the median (quartile). MCA: middle cerebral arteries; ACA: anterior cerebral artery; ICA: internal carotid arteries; $\mathrm{Pl}$ : pulsation index;RI: resistance index

\subsubsection{Correlation analysis of TCD data and DTI parameters}

Analysing the DTI data of the three ROI (ACC, PFC and basal ganglia), the DTI data in basal ganglia showed being associated with the PI and RI of MCA and ICA(Table4). FA skeleton negatively correlated with $\mathrm{PI}$ and RI of MCA and ICA, MD skeleton, both with $\mathrm{AD}$ and RD skeleton, showed positively correlating with $\mathrm{PI}$ and $\mathrm{RI}$ of MCA and ICA $(\mathrm{P}<0.01)$. As to the PFC and ACC, FA skeleton still showed negatively correlating with $\mathrm{PI}$ and $\mathrm{RI}$ of $\mathrm{ACA}$ and ICA, otherwise, MD skeleton and RD skeleton positively correlated with the PI and RI of ACA and ICA(Table5,6).

Table 4:Correlation analysis of basal ganglia DTI parameters and TCD data

\begin{tabular}{|c|c|c|c|c|c|c|c|c|}
\hline & \multicolumn{2}{|c|}{$\begin{array}{l}\text { Basal ganglia } \\
\text { FA skeleton }\end{array}$} & \multicolumn{2}{|c|}{$\begin{array}{l}\text { Basal } \\
\text { ganglia MD skeleton }\end{array}$} & \multicolumn{2}{|c|}{$\begin{array}{l}\text { Basal ganglia } \\
\text { AD skeleton }\end{array}$} & \multicolumn{2}{|c|}{$\begin{array}{l}\text { Basal } \\
\text { ganglia RD skeleton }\end{array}$} \\
\hline & $\mathrm{R}$ & $\mathrm{P}$ & $\mathrm{R}$ & $P$ & $\mathrm{R}$ & $P$ & $\mathrm{R}$ & $P$ \\
\hline $\begin{array}{l}\text { Left ICA } \\
\text { terminal end } \\
\text { PI }\end{array}$ & -0.678 & 0.001 & 0.683 & 0.001 & 0.549 & 0.012 & 0.728 & $<0.001$ \\
\hline $\begin{array}{l}\text { Left ICA } \\
\text { terminal end } \\
\text { RI }\end{array}$ & -0.658 & 0.002 & 0.698 & 0.001 & 0.588 & 0.006 & 0.737 & $<0.001$ \\
\hline Left MCA RI & -0.631 & 0.003 & 0.639 & 0.002 & 0.459 & 0.042 & 0.692 & 0.001 \\
\hline Left MCA PI & -0.592 & 0.006 & 0.604 & 0.005 & 0.425 & 0.062 & 0.654 & 0.002 \\
\hline Right MCA PI & -0.58 & 0.007 & 0.743 & $<0.001$ & 0.658 & 0.002 & 0.723 & $<0.001$ \\
\hline Right MCA RI & -0.601 & 0.005 & 0.757 & $<0.001$ & 0.668 & 0.001 & 0.742 & $<0.001$ \\
\hline $\begin{array}{l}\text { Right ICA } \\
\text { terminal end } \\
\text { PI }\end{array}$ & -0.591 & 0.006 & 0.751 & $<0.001$ & 0.614 & 0.004 & 0.744 & $<0.001$ \\
\hline $\begin{array}{l}\text { Right ICA } \\
\text { terminal end } \\
\text { RI }\end{array}$ & -0.59 & 0.006 & 0.76 & $<0.001$ & 0.635 & 0.003 & 0.747 & $<0.001$ \\
\hline
\end{tabular}

FA: Fractional anisotropy; MD: average diffusivity; RD: radial diffusivity; AD: axial diffusivity; MCA: middle cerebral arteries; ICA: internal carotid arteries; PI: pulsation index; RI: resistance index.

Table 5:Correlation analysis of PFC DTI parameters and TCD data 


\begin{tabular}{|lllllll|}
\hline & \multicolumn{2}{l}{ PFC FA skeleton } & \multicolumn{2}{l}{ PFC MD skeleton } & \multicolumn{2}{l|}{ PFC RD skeleton } \\
\hline & $\mathrm{R}$ & $\mathrm{P}$ & $\mathrm{R}$ & $\mathrm{P}$ & $\mathrm{R}$ & $\mathrm{P}$ \\
\hline Left ICA terminal end PI & -0.653 & 0.002 & 0.49 & 0.028 & 0.515 & 0.02 \\
\hline Left ICA terminal end RI & -0.655 & 0.002 & 0.483 & 0.031 & 0.512 & 0.021 \\
\hline Left ACA PI & -0.509 & 0.022 & 0.536 & 0.015 & 0.501 & 0.025 \\
\hline Left ACA RI & -0.513 & 0.021 & 0.536 & 0.015 & 0.501 & 0.025 \\
\hline Right ACA PI & -0.563 & 0.01 & 0.531 & 0.016 & 0.49 & 0.028 \\
\hline Right ACA RI & -0.556 & 0.011 & 0.523 & 0.018 & 0.481 & 0.032 \\
\hline Right ICA terminal end PI & -0.659 & 0.002 & 0.573 & 0.008 & 0.577 & 0.008 \\
\hline Right ICA terminal end RI & -0.658 & 0.002 & 0.569 & 0.009 & 0.58 & 0.007 \\
\hline
\end{tabular}

PFC: prefrontal cortex; FA: Fractional anisotropy; MD: average diffusivity; RD: radial diffusivity; ACA: anterior cerebral artery; ICA: internal carotid arteries; PI: pulsation index; RI: resistance index

\section{Table 6:Correlation analysis of ACC DTI parameters and TCD data}

\begin{tabular}{|lllllll|}
\hline & \multicolumn{2}{c}{ ACC FA skeleton } & \multicolumn{2}{l}{ ACC MD skeleton } & \multicolumn{2}{l|}{ ACC RD skeleton } \\
\hline & $\mathrm{R}$ & $\mathrm{P}$ & $\mathrm{R}$ & $\mathrm{P}$ & $\mathrm{R}$ & $\mathrm{P}$ \\
\hline Left ICA terminal end PI & -0.477 & 0.034 & 0.655 & 0.002 & 0.6 & 0.005 \\
\hline Left ICA terminal end RI & -0.525 & 0.017 & 0.696 & 0.001 & 0.637 & 0.003 \\
\hline Left ACA PI & -0.493 & 0.027 & 0.543 & 0.013 & 0.514 & 0.02 \\
\hline Left ACA RI & -0.495 & 0.026 & 0.55 & 0.012 & 0.519 & 0.019 \\
\hline Right ACA PI & -0.473 & 0.035 & 0.521 & 0.018 & 0.49 & 0.028 \\
\hline Right ACA RI & -0.468 & 0.037 & 0.526 & 0.017 & 0.491 & 0.028 \\
\hline Right ICA terminal end PI & -0.557 & 0.011 & 0.69 & 0.001 & 0.62 & 0.004 \\
\hline Right ICA terminal end RI & -0.56 & 0.01 & 0.695 & 0.001 & 0.625 & 0.003 \\
\hline
\end{tabular}

ACC: anterior cerebral artery; FA: Fractional anisotropy; MD: average diffusivity; RD: radial diffusivity; ACA: anterior cerebral artery; ICA: internal carotid arteries; PI: pulsation index; RI: resistance index

3.2 Analysis of PD-related pain in the patients

3.2.1 Clinical features of patients 
The patients were classified as the group with PD-related pain and the group without PD-related pain by use of criteria of Ford classification method ${ }^{[6]}$. The patients with PD-related pain complained burning pain, acupuncture sensation, tightening sensation, knife cutting sensation. The patients were unable to identify the pain area or the pain area vacillates. Patients with PD-related pain $(\mathrm{N}=6,30 \%)$ and patients without PD-related pain $(N=14,70 \%)$ are closely matched in age ( $65 \pm 8.94$ vs. $63.71 \pm 10.54, p=0.798)$. No significant differences in score of MDS-UPDRS $\triangle$ and III either in "off" or "on" medication states between patients with or without PD-related pain. Again, there were no statistical difference in levodopa dose, HAMA, HAMD, age and disease duration. However, there was a statistically significant difference in PSQI scores $(P<0.05)$ (Table7). Moreover, it showed no correlation between posture/balance disorder and PDrelated pain using Chi-square test $(\mathrm{P}=1)$.

\section{Table 7: Comparison of clinical information between patients with and without PD-related pain}

\begin{tabular}{|lllll|}
\hline & PD-related pain & Without PD-related pain & T/Z & $P$ \\
\hline Levodopa Dose & $291.67 \pm 182.8$ & $307.14 \pm 204.62$ & 0.16 & 0.875 \\
\hline PSQI & $11.67 \pm 5.28$ & $5.92 \pm 3.45$ & -2.856 & 0.011 \\
\hline HAMA & $15.5(2.25-38.25)$ & $6(1.75-16.25)$ & -1.116 & 0.264 \\
\hline HAMD & $15.50 \pm 10.82$ & $10.21 \pm 9.01$ & -1.135 & 0.271 \\
\hline UPDRS $\rrbracket$ & $4.5(3.75-15)$ & $10(5.75-14.5)$ & -1.283 & 0.2 \\
\hline UPDRS $\square$ & $22.33 \pm 9.56$ & $25.78 \pm 10.79$ & 0.676 & 0.507 \\
\hline Age & $65.00 \pm 8.94$ & $63.71 \pm 10.54$ & -0.26 & 0.798 \\
\hline Disease duration & $6.67 \pm 2.88$ & $5.79 \pm 3.02$ & -0.606 & 0.552 \\
\hline
\end{tabular}

The value of data that conforms to the normal distribution is displayed as the mean \pm standard deviation, and the value of the data that does not conform to the normal distribution is displayed as the median (quartile). HAMA: Hamilton Anxiety Scale; HAMD: Hamilton Depression Scale; PSQI: Pittsburgh sleep quality index.

\subsubsection{Analysis on fiber bundle tracking}

The fiber bundles were tracked from midbrain to the basal ganglia and PFC.The average FA value of the tracked fiber bundle from the midbrain to basal ganglia was significantly higher in the patients with PDrelated pain than that in patients without PD-related pain. Moreover, there were more fibers from midbrain to basal ganglia in patients without PD-related pain $(\mathrm{p}<0.05)$ (Table8 and Fig.2-A). No statistic difference were found in fibers amount from midbrain to PFC between the two groups, they all showed less fibers left there(Fig.2-B). 
Analysis of the white matter fiber bundles in the locus coeruleus area showed that there were no significant differences in the number of fiber bundles, the maximum FA value and the maximum fiber length between the two groups( $p>0.05)$ (Table 8 and Fig.3).

Table 8:The difference between the groups in fiber bundle tracking

\begin{tabular}{|lllll|}
\hline & $\begin{array}{l}\text { With PD- } \\
\text { related pain }\end{array}$ & $\begin{array}{l}\text { Without PD- } \\
\text { related pain }\end{array}$ & T/Z & $P$ \\
\hline Number of midbrain to basal ganglia fibers & $\begin{array}{l}176.5(71.75- \\
339)\end{array}$ & $30(6.5-93.5)$ & -2.187 & 0.029 \\
\hline $\begin{array}{l}\text { Average fiber length from midbrain to basal } \\
\text { ganglia }\end{array}$ & $\begin{array}{l}81.2886(67.91- \\
87.9)\end{array}$ & $\begin{array}{l}53.2199(26.52- \\
84.18)\end{array}$ & -1.32 & 0.187 \\
\hline $\begin{array}{l}\text { Average FA value of midbrain to basal } \\
\text { ganglia fibers }\end{array}$ & $\begin{array}{l}0.4947(0.47- \\
0.51)\end{array}$ & $\begin{array}{l}0.42265(0.31- \\
0.47)\end{array}$ & -2.434 & 0.015 \\
\hline $\begin{array}{l}\text { Number of fiber bundles passing through } \\
\text { locus coeruleus }\end{array}$ & $111.5 \pm 23.72$ & $105.6 \pm 40.4$ & -0.333 & 0.743 \\
\hline $\begin{array}{l}\text { The maximum FA value of the fiber bundle } \\
\text { walking in the locus coeruleus nucleus }\end{array}$ & $0.614 \pm 0.074$ & $0.6 \pm 0.039$ & -0.566 & 0.578 \\
\hline $\begin{array}{l}\text { Maximum length of fiber bundles running in } \\
\text { the locus coeruleus nucleus }\end{array}$ & $83.59 \pm 27.75$ & $70.98 \pm 22.57$ & -1.071 & 0.298 \\
\hline
\end{tabular}

\section{Discussion}

Studies have shown that patients with PIGD Parkinson's disease are more likely to have difficulty walking independently, more dependent on caregivers, and more cognitive decline, with a greater risk of disability. Therefore, in this study, the posture and balance in PD are particularly important. In our study, we performed investigation of risk factors of posture/balance disorder from clinical feature to DTI fibers tracking and parameters of cerebral blood flow. In our study, patients with higher posture/balance scores had higher UPDRS scores and more levodopa use, indicating that the more severe the disease, the worse the posture and balance. Ali Falaki and colleagues found that dopamine-replacement drugs lead to higher indices of multi-muscle synergies and higher anticipatory synergy adjustments indices in $\mathrm{PD}^{[7]}$. In addition, although levodopa improves certain aspects of motor function, it increases sway and destroys the sensory organization of posture control ${ }^{[8,9]}$. This study confirmed previous results and showed that the posture/balance disorder group does not seem to have sufficient sensorimotor control to benefit from the reduced stiffness and improved axial posture provided by levodopa, which instead increases the incidence of posture/balance disorder. This will seriously affect the lives of patients.

Previous research on DTI have revealed that PIGD patients had greater diffusivity and greater reduction of white matter integrity in basal ganglia ${ }^{[10]}$, as well, the result of functional Near Infrared Spectrum Instrument indicates that PFC is involved in the maintenance of attention-demanding balance tasks ${ }^{[11]}$. In our study on DTI, instability, FA was lower in basal ganglia, PFC and ACC in the patients with more severe 
posture, these changes were driven by an increase in MD, AD and RD, suggesting that demyelination and changes in axon integrity in these areas are related to posture/balance disorder. They are similar to previous studies on instability, although our patients were not belong to the patients of PIGD in clinical feature. The correlation of posture/instability with ACC in our study, in fact, consistent with previous studies that improving posture/balance disorder was related to changes in the gray matter of the bilateral $\mathrm{ACC}^{[4]}$. The basal ganglia circuit is important for the stability of multiple muscle movements and the agility of behavior. PFC and ACC participate in the allocation of attention resources to maintain posture contro [ ${ }^{[12]}$, there are a wide range of transmitter nerve fibers in these brain areas. Posture/balance disorder could not be adequately improved by levodopa, which means that the non-dopaminergic pathway plays roles $^{[13,14]}$. Previous studies have suggested that the cholinergic system and defective adrenergic innervation are suspected to contribute to the postural control of PD. It is reported that PFC, ACC and brain regions have a large number of adrenergic nerve fibers projected from the locus coeruleus. This led us to consider the role of the norepinephrine system in posture/balance disorder. But, we are not sure whether the neurotransmitter of the fibers in these areas are dopamine or norepinephrine, more targeted studies are needed to explore this.

It is believed that cerebral ischemia contributes to the brain damage in PD. We analyzing the correlation of TCD with posture/balance disorder and DTI fibers on ROI. The results showed that the poor posture group had higher scores in RI and PI values of ICA system vessels. It gives us a suggestion that blood vessels have poor function in this group. And then, FA skeleton negatively correlated with $\mathrm{PI}$ and $\mathrm{RI}$ of ACA, MCA and ICA, as corresponding, MD, AD and RD skeleton showed positive correlation. The relationship of these three aspects strongly suggests that patients with unstable posture have more severe brain fiber damage and poorer cerebrovascular function, and cerebral ischemia has contributions to this pathological state and clinical manifestations.

PD patients with Pisa syndrome suffered more chronic pain than patients with normal posture. It may due to the continuous muscle stiffness and dystonia. Patients with posture and balance dysfunction may give the greater efforts of muscle construction in daily activities. But patients have pain with variable positions, it suggests that the causes of pain may be complex, and not just muscle and fascia strain. The analysis of our patients found that PD-related pain was positively correlated with sleep score, indicating that patients with sleep disorders were more likely to have chronic pain. Acute sleep deprivation has been reported to amplify pain responses in the primary somatosensory cortex. That could be one reason for the correlation. Both pain and sleep disturbances are known to involve brainstem structures and changes in neurotransmitter systems (Dopamine and Norepinephrine) ${ }^{[15]}$, we tracked the fiber bundles from the midbrain to the basal ganglia and PFC, and calculated the number of fiber bundles running at the locus coeruleus and their maximum FA value and length. We found that patients with PDrelated pain have an increase in the fiber projection from the midbrain to the basal ganglia compared with those patients without PD-related pain. There was no difference in the comparison of the fiber bundles in locus coeruleus between the two groups. And we think about this in detail, what is the role of these projection fibers in pain patients? What are the properties of the fibers, and what are the mechanisms that 
cause them? There was no difference in UPDRS motor function scores between patients with and without pain, considering that the severity of the disease was not the cause of the pain, and no reduction in projected fibers confirmed that the pathological manifestations in the brain were not worse. Thus, it is possible that some abnormal regulatory fiber amplifies the sensation of pain, and that the improper proliferation of such regulatory fiber makes the patient misunderstand pain. It was reported that the part of calcitonin gene-related peptide immunopositive neurons in the lateral parabrachial nucleus send projection fibers to both the central amygdaloid nucleus and ventral tegmental area by the way of axon collaterals, which are activated by the nociceptive stimulation in the spared nerve injury condition ${ }^{[16]}$. Is it compensatory hyperplasia of this fiberor dysplasia, or is there a relative increase of this fiber due to a decrease in other fibers, that causes Parkinson's disease pain? This is an interesting finding, and of course we need to expand the sample size and analyze it carefully to confirm the validity of this phenomenon.

The main advantage of this study is the application of DTI technology combined with TCD to clarify the relationship between white matter damage and posture/balance disorder in the state of cerebral ischemia, and it is surprising to find that although PD-related pain has nothing to do with Posture/balance, there is an abnormal increase in fibers from the midbrain to the basal ganglia, which is worthy of further exploration. We also admit certain limitations. Due to factors such as poor image quality, this study excluded patients who did not meet the conditions, resulting in a small sample size for the analysis. Therefore, our research results need to be confirmed in a larger sample.

\section{Conclusion}

Posture and balance in PD were correlated with the severity of the disease and the dosage of compound levodopa. Posture and balance in Parkinson's disease were related to changes in the white matter integrity of the PFC,ACC, and basal ganglia. The function of cerebral arteries had contributions to white matter integrity of these area and posture/balance. PD-related pain was positively correlated with sleep score. Patients with PD-related pain had an increase in the fiber projection from the midbrain to the basal ganglia. No relation was found between posture/balance disorder with PD-related pain.

\section{Abbreviations}

TCD: transcranial Doppler; PIGD: postural instability/gait difficulty; PD:Parkinson's disease; MMSE: MiniMental State Examination; HAMA: Hamilton Anxiety Scale; HAMD: Hamilton Depression Scale; PSQI: Pittsburgh sleep quality index; MCA: middle cerebral arteries; ACA: anterior cerebral artery; ICA: internal carotid arteries; Pl: pulsation index; RI: resistance index; FA: Fractional anisotropy; MD: average diffusivity; RD: radial diffusivity; AD: axial diffusivity;

\section{Declarations}

Acknowledgements 
All authors would appreciate their inputs from the team of magnetic resonance imaging chamber and cerebral blood flow chamber in the Second Affiliated Hospital at Harbin Medical University, China on the data collecting

\section{Authors' contributions}

Xiaoying Wang: writing-original draf, acquisition of data, interpretation and analysis of data.

Yingying Zhang and Qing Gao: interpretation and analysis of data.

Wenhui Guo and Dan Liu: collection of data

Limei Zhang: design of the study, interpretation and analysis of data, supervision, funding acquisition.

Kuang Fu: design of the study, supervision.

All authors reviewed the manuscript.

\section{Funding}

This work was supported by the National Natural Science Foundation of China (Grant No.81871003).

\section{Ethics approval and consent to participate}

The process of the study was approved by the Ethics Committee of the Second Affiliated Hospital of Harbin Medical University (approval number: K1/2018-230), in compliance with the Declaration of Helsinke.

\section{Consent for publication}

Not applicable

\section{Competing interests}

The authors declare that they have no competing interests.

\section{Availability of data and materials}

The datasets used and/or analyzed during the current study are available from the corresponding authors upon reasonable request.

\section{References}

1. Latt M D, Lord S R, Morris J G, et al. Clinical and physiological assessments for elucidating falls risk in Parkinson's disease[J]. Mov Disord, 2009, 24(9): 1280-9. DOI:10.1002/mds.22561 
2. Forsyth A L, Joshi R Y, Canning C G, et al. Flexed Posture in Parkinson Disease: Associations With Nonmotor Impairments and Activity Limitations[J]. Phys Ther, 2019, 99(7): 893-903.

DOI:10.1093/ptj/pzz033

3. Lenfeldt $\mathrm{N}$, Holmlund $\mathrm{H}$, Larsson $\mathrm{A}$, et al. Frontal white matter injuries predestine gait difficulties in Parkinson's disease[J]. Acta Neurol Scand, 2016, 134(3): 210-8. DOI:10.1111/ane.12532

4. Goel R, Nakagome S, Rao N, et al. Fronto-Parietal Brain Areas Contribute to the Online Control of Posture during a Continuous Balance Task[J]. Neuroscience, 2019, 413: 135-153.

DOI:10.1016/j.neuroscience.2019.05.063

5. Jahn A, Nee D E, Alexander W H, et al. Distinct Regions within Medial Prefrontal Cortex Process Pain and Cognition[J]. J Neurosci, 2016, 36(49): 12385-12392. DOI:10.1523/JNEUROSCI.2180-16.2016

6. Ford B. Pain in Parkinson's disease[J]. Mov Disord, 2010, 25 Suppl 1: S98-103. DOI:10.1002/mds.22716

7. Falaki A, Huang X, Lewis M M, et al. Dopaminergic modulation of multi-muscle synergies in postural tasks performed by patients with Parkinson's disease[J]. J Electromyogr Kinesiol, 2017, 33: 20-26. DOI:10.1016/j.jelekin.2017.01.002

8. Pelicioni P H S, Brodie M A, Latt M D, et al. Head and trunk stability during gait before and after levodopa intake in Parkinson's disease subtypes[J]. Exp Gerontol, 2018, 111: 78-85.

DOI:10.1016/j.exger.2018.06.031

9. Bonnet C T, Delval A, Szaffarczyk S, et al. Levodopa has primarily negative influences on postural control in patients with Parkinson's disease[J]. Behav Brain Res, 2017, 331: 67-75.

DOI:10.1016/j.bbr.2017.05.033

10. Nagae L M, Honce J M, Tanabe J, et al. Microstructural Changes within the Basal Ganglia Differ between Parkinson Disease Subtypes[J]. Front Neuroanat, 2016, 10: 17.

DOI:10.3389/fnana.2016.00017

11. Mahoney J R, Holtzer R, Izzetoglu M, et al. The role of prefrontal cortex during postural control in Parkinsonian syndromes a functional near-infrared spectroscopy study[J]. Brain Res, 2016, 1633: 126-138. DOI:10.1016/j.brainres.2015.10.053

12. Tan S Y Z, Keong N C H, Selvan R M P, et al. Periventricular White Matter Abnormalities on Diffusion Tensor Imaging of Postural Instability Gait Disorder Parkinsonism[J]. AJNR Am J Neuroradiol, 2019, 40(4): 609-613. DOI:10.3174/ajnr.A5993

13. Crouse J J, Phillips J R, Jahanshahi M, et al. Postural instability and falls in Parkinson's disease[J]. Rev Neurosci, 2016, 27(5): 549-55. DOI:10.1515/revneuro-2016-0002

14. Di Giulio I, St George R J, Kalliolia E, et al. Maintaining balance against force perturbations: impaired mechanisms unresponsive to levodopa in Parkinson's disease[J]. J Neurophysiol, 2016, 116(2): 493502. DOI:10.1152/jn.00996.2015

15. Vila-Cha N, Cavaco S, Mendes A, et al. Sleep disturbances in Parkinson's disease are associated with central parkinsonian pain[J]. J Pain Res, 2019, 12: 2137-2144. DOI:10.2147/JPR.S206182 
16. Qiao Y, Zhang C K, Li Z H, et al. Collateral Projections from the Lateral Parabrachial Nucleus to the Central Amygdaloid Nucleus and the Ventral Tegmental Area in the Rat[J]. Anat Rec (Hoboken), 2019, 302(7): 1178-1186. DOI:10.1002/ar.23983

\section{Figures}

\section{basal ganglia}

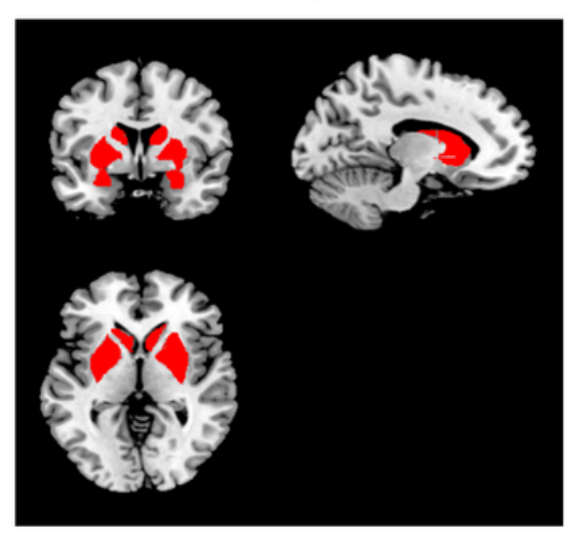

ACC

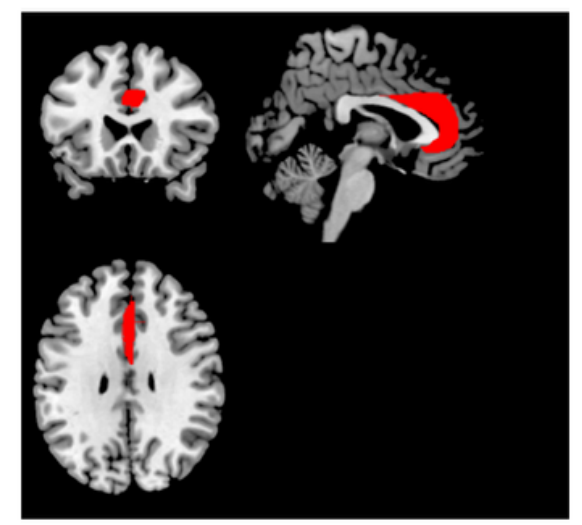

PFC

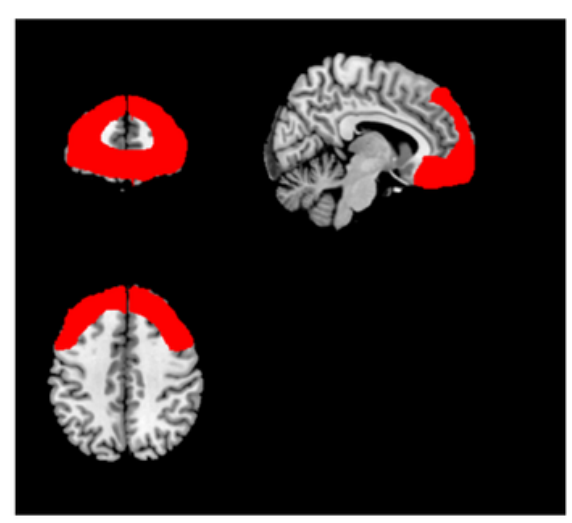

Figure 1

Extract the brain area of interest according to the brain atlas(PFC: prefrontal cortex; ACC: anterior cingulate cortex)

with PD-related pain
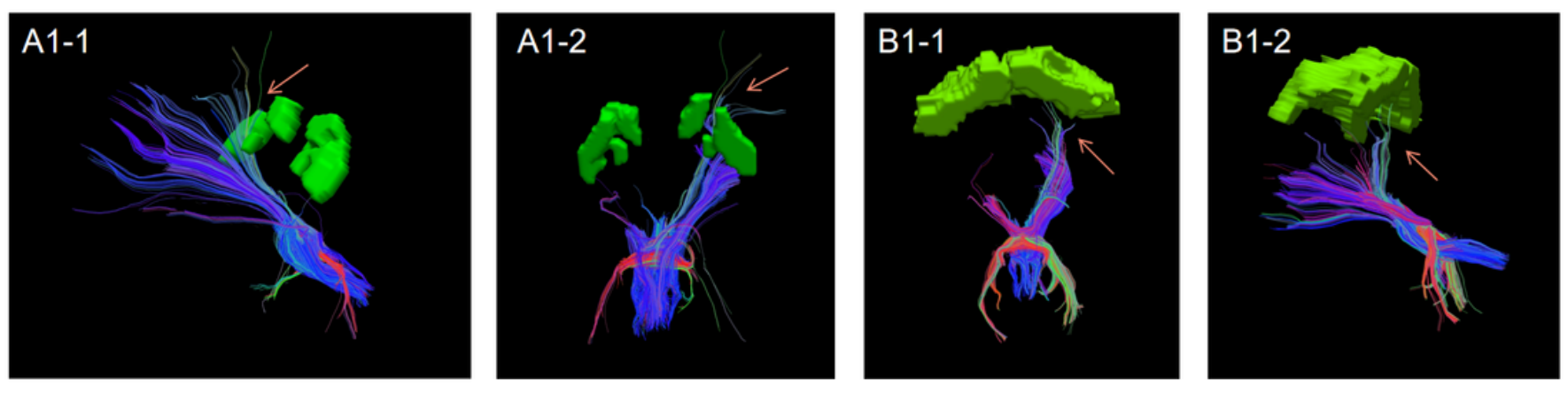

without PD-related pain
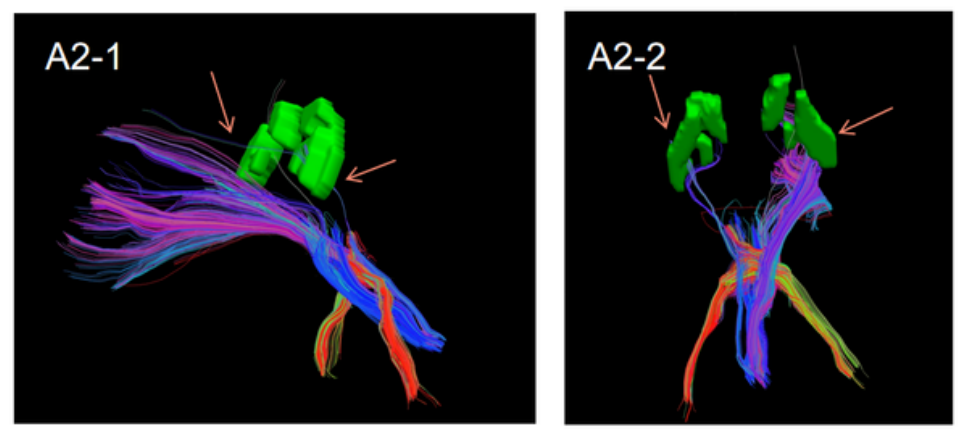
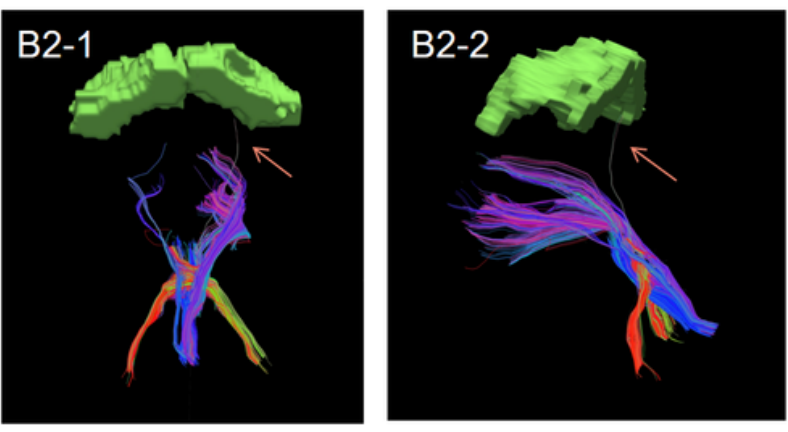
Figure 2

DTI display of different groups with and without PD-related pain. The red arrow indicates the fiber bundle from the midbrain to a specific brain area.A1 and A2 show the midbrain to the basal ganglia; B1 and B2 show the midbrain to the PFC

\section{with PD-related pain}
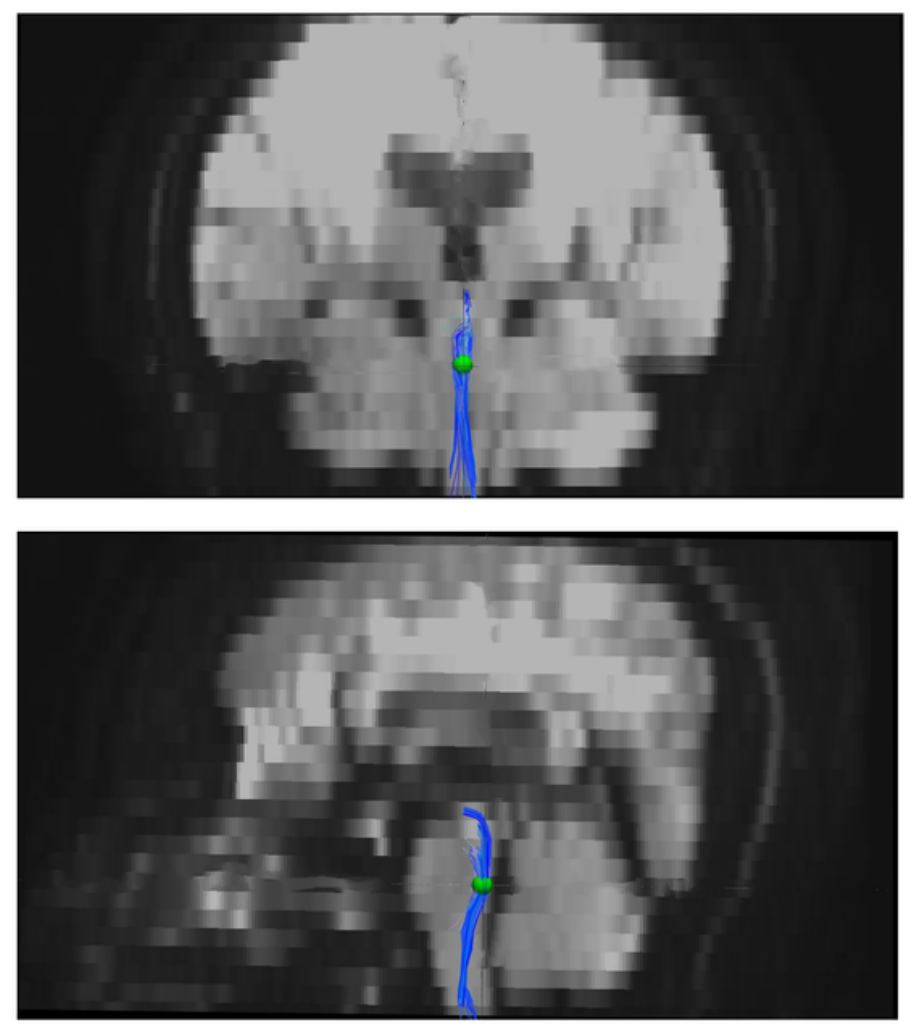

without PD-related pain
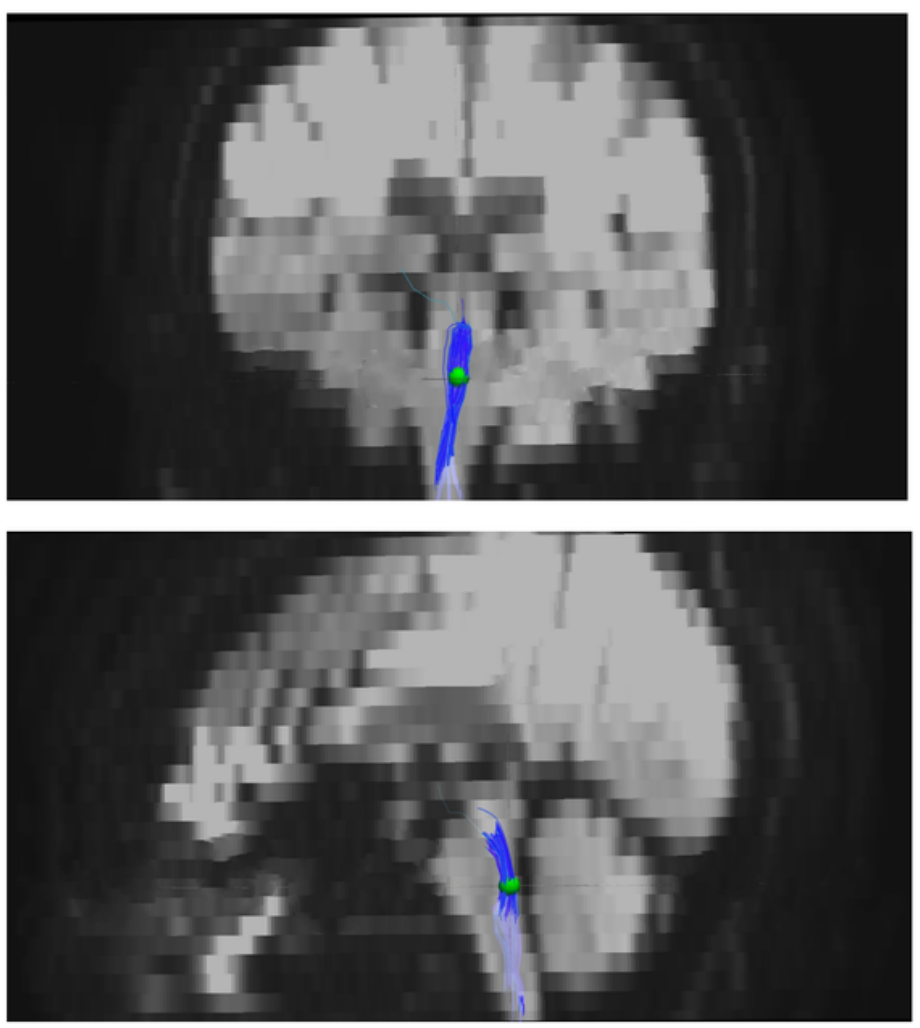

\section{Figure 3}

The display of fiber bundles passing through the locus coeruleus in patients with or without PD-related pain. 